Nervenarzt 2020 $91: 227-232$

https://doi.org/10.1007/s00115-019-00795-x

Online publiziert: 29. August 2019

(c) Der/die Autor(en) 2019

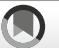

Check for
updates

\author{
Charlotte Pervilhac ${ }^{1,2} \cdot$ Kyrill Schoilew $^{3} \cdot$ Hansjörg Znoj ${ }^{2} \cdot$ Thomas J. Müller $^{1,3}$ \\ ${ }^{1}$ Privatklinik Meiringen, Willigen, Meiringen, Schweiz \\ ${ }^{2}$ Institut für Psychologie, Abteilung für Gesundheitspsychologie und Verhaltensmedizin, Universität Bern, \\ Bern, Schweiz \\ ${ }^{3}$ Universitätsklinik für Psychiatrie und Psychotherapie, Zentrum für translationale Psychiatrie (TRC), Bern, \\ Schweiz
}

\title{
Wetter und Suizid
}

\section{Assoziation zwischen meteorologischen Variablen und suizidalem Verhalten - eine qualitative systematische Übersichtsarbeit}

saisonalen Effekten deutlich: So zeigen sich sowohl in nördlicher als auch in südlicher Hemisphäre saisonale Muster für Suizide, die im Allgemeinen mit einem Anstieg während der Frühlingsmonate und im frühen Sommer festzustellen sind (vgl. $[1,9,10]$ für eine Übersicht zur Thematik). Überdies wird bereits seit Anfang des 20. Jahrhunderts der Einfluss einzelner Wetterfaktoren auf Suizidalität empirisch untersucht (vgl. [14]). Meteorologische Variablen werden in der Wissenschaft seither als mögliche externe Stressoren diskutiert, die suizidales Verhalten bei vulnerablen Personen begünstigen könnten [21, 50, 52, 54]. $\mathrm{Zu}$ möglichen neurobiologischen Effekten von Wetter auf das psychische Wohlbefinden finden sich bislang lediglich vereinzelt Erklärungsansätze [8, 23, 29, 47, 48]. Einhergehend mit den weitreichenden Implikationen aktueller sowie künftig zu erwartender Auswirkungen des globalen Klimawandels auf die menschliche Gesundheit [4, $11,30,31$ ] rückt der spezifische Einfluss einzelner Wettervariablen als potenzielle Risikofaktoren zunehmend in den Fokus der Suizidforschung.

Die vorliegende Übersichtsarbeit bietet einen fundierten Einblick in den aktuellen Forschungs- und Wissensstand zum Einfluss meteorologischer Variablen auf suizidales Verhalten.

\section{Material und Methoden}

Zur Erfassung relevanter Studien wurde eine kriterienbasierte, systematische Literaturrecherche nach den Preferred Reporting Items for Systematic Reviews and Meta-Analyses (PRISMA)-Kriterien durchgeführt [32]. In der MEDLINEDatenbank (PubMed, National Library of Medicine) wurde am 31.01.2019 folgender Suchalgorithmus unter Anwendung von Medical Subject Headings (MeSH) sowie weiterer relevanter Suchwörter eingesetzt: ("Suicide" [MeSH] OR "Suicid $\left.{ }^{* \prime}\right)$ AND ("Weather" ${ }^{\prime \prime}[\mathrm{MeSH}]$ OR"Climate" [MeSH] OR" Atmospheric Pressure" $[\mathrm{MeSH}]$ OR "Sunlight" OR "Heat" OR "Temperature" OR "Wind" OR "Rain" OR "Lightning" OR "Thunder $^{* \prime}$ OR "Humidity" OR "Cloud*" OR "Fog" OR "Snow" OR "Weather" OR "Meteorological ${ }^{\star \prime}$ OR "Season ${ }^{* \prime}$ ) AND (English[Language] OR German[Language]).

Die Suche wurde mithilfe gängiger Internetsuchmaschinen (z. B. Google Scholar) vervollständigt. Identifizierte Übersichtsarbeiten wurden durchgesehen und mit dem Rechercheergebnis verglichen. Eingeschlossen wurden englisch- und deutschsprachige Originalarbeiten mit Peer-Review ohne zeitliche Limitationen. Die Referenzen der ausgewählten Arbeiten wurden ebenso nach relevanten Studien durchsucht. Der Fokus lag auf Einschluss von Studien, die den Einfluss meteorologischer Fakto- 


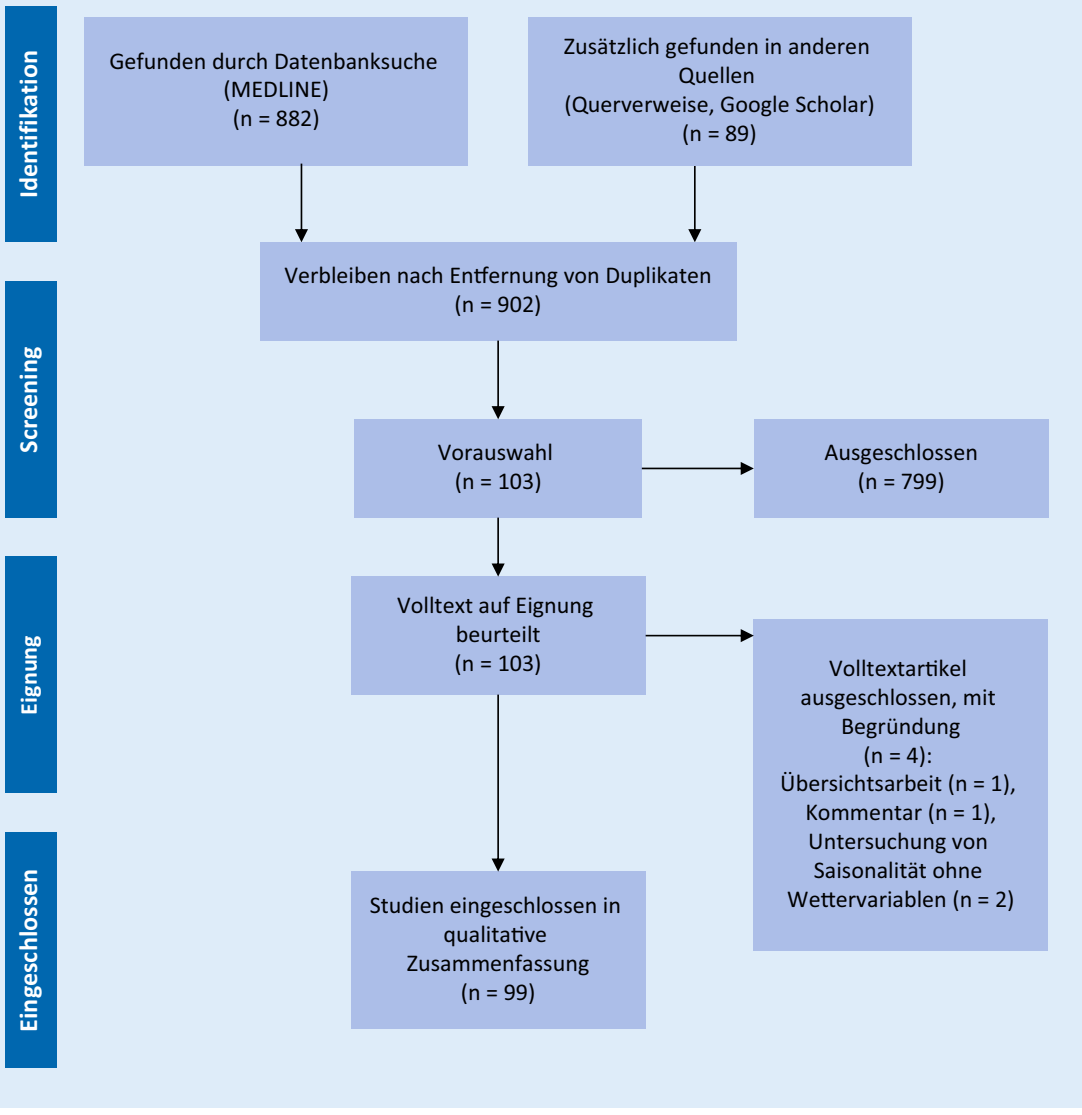

Abb. 1 ॥ PRISMA-Flussdiagramm der unterschiedlichen Phasen einer systematischen Übersichtsarbeit

ren auf suizidale Handlungen (Suizide und Suizidversuche) untersuchen. Reine Zeitreihenanalysen zum Einfluss von Saisonalität ohne Berücksichtigung von Wettervariablen sowie Untersuchungen zum Einfluss von Naturkatastrophen auf suizidale Handlungen wurden ausgeschlossen.

\section{Ergebnisse}

Insgesamt wurden 971 Abstracts identifiziert. Nach Entfernung von Duplikationen, Sichtung der Abstracts und Ausschluss nicht zur Fragestellung gehörender Arbeiten, wurden 103 Studien in die Vorauswahl aufgenommen (• Abb. 1, PRISMA-Flussdiagramm). 799 Arbeiten wurden aufgrund von Mehrfachpublikation, nicht näher spezifizierter Studiendesigns und nicht verwertbarer Daten ausgeschlossen. Der Volltext von 103 Arbeiten wurde auf Eignung beurteilt. Von diesen Untersuchungen wurden weitere 4 Arbeiten ausgeschlossen, da es sich hierbei um eine Übersichtsarbeit, ein Kommentar und Zeitreihenanalysen ohne Wettervariablen gehandelt hat. Insgesamt konnten 99 relevante Studien identifiziert werden.

\section{Studiendesign}

Es zeigten sich erhebliche Unterschiede in den verwendeten Studiendesigns: Die Größe des Untersuchungsgebietes reichte von einzelnen regionalen, nationalen bis hin zu multinationalen Betrachtungen, die 62 Nationen [26] umfassten. Vornehmlich waren Industrienationen Gegenstand der Analysen. Es fanden sich Länder verschiedener Kontinente, lediglich Staaten des afrikanischen Kontinents waren nicht vertreten. Der Beobachtungszeitraum reichte von einem Jahr bis zu 258 Jahren [22]. Es wurden entweder Suizidversuche $(n=9)$, Suizide $(n=76)$ oder beide Parameter $(n=14)$ berücksichtigt. Hierbei umfasste die Anzahl der Ereignisse im jeweiligen
Beobachtungszeitraum $n=73$ [20] bis $n=501.950$ [27]. 24 Studien waren hier$\mathrm{zu}$ keine Angaben zu entnehmen. Die untersuchten meteorologischen Parameter unterschieden sich sowohl in Anzahl als auch Art der Variablen erheblich voneinander. Am häufigsten fanden die Variablen Temperatur, Niederschlag, Luftfeuchtigkeit, Sonnenstunden und Luftdruck Berücksichtigung. Weiterhin zeigten sich deutliche Unterschiede in der statistischen Auswertung. Mögliche zusätzliche Einflussfaktoren, wie etwa saisonale Effekte, Soziodemographie und Suizidmethoden (harte vs. weiche Methoden), fanden lediglich vereinzelt Berücksichtigung. Insbesondere im Hinblick auf die Zeitspanne der untersuchten Variablen fielen erhebliche Unterschiede auf. Es fanden sich Analysen, in denen Wettervariablen und/oder Suizidraten auf Grundlage von Tages-, Wochen-, Monats- und Jahresdaten korreliert wurden. Cluster- und Lag-Effekte wurden lediglich vereinzelt berechnet.

Kernparameter und Hauptergebnisse der identifizierten Studien wurden dem jeweiligen Studiendesign entsprechend auf Basis von Tages- (Zusatzmaterial online: Tab. 1), Wochen- (Zusatzmaterial online: Tab. 2), Monats- (Zusatzmaterial online: Tab. 3) und Jahresdaten (Zusatzmaterial online: Tab. 4) zusammengefasst.

\section{Hauptergebnisse}

Die Mehrzahl der eingeschlossenen Studien zeigte signifikante Assoziationen zwischen mindestens einer Wettervariable und Suizidalität. Von den 99 eingeschlossenen Studien zeigten sichlediglich in 17 Untersuchungen keine signifikanten Assoziationen [7, 15, 16, 18, 20, 24, $35,36,38,40,41,43,44,49,51,55$, 57]. Im Allgemeinen waren inkonsistente und widersprüchliche Ergebnisse festzustellen. Am häufigsten fanden sich positive Korrelationen zwischen Temperatur und Suizidalität $(n=49)$, wobei sich auch hier vereinzelt negative Korrelationen $(n=10)$ oder nicht signifikante Ergebnisse zeigten $(n=25)$. 


\section{Diskussion}

Die vorliegende Übersichtsarbeit fasst die aktuelle Evidenz zu Assoziationen zwischen meteorologischen Variablen und suizidalem Verhalten zusammen. Sie beruht auf einer systematischen Literaturrecherche, die eine umfassende Identifikation relevanter Studien ermöglichen soll. Zugleich wirkt der strenge kriterienbasierte Ansatz jedoch limitierend: Einerseits bleiben Publikationen außerhalb der MEDLINE-Datenbank weitgehend unberücksichtigt. Andererseits wurden lediglich englisch- und deutschsprachige Originalarbeiten eingeschlossen; weitere internationale Veröffentlichungen (so sind etwa auch französischsprachige Veröffentlichungen auszumachen - vgl. etwa $[5,25,33])$ und alternative Publikationsformen (Dissertationen, Monographien, Kongressbeiträge etc.) wurden nicht einbezogen.

Zuletzt wurde im Jahre 2003 eine systematische Übersichtsarbeit zur Thematik publiziert [13]. Während vor über 15 Jahren noch 27 Studien von Deisenhammer eingeschlossen wurden, sind nunmehr 99 Arbeiten auszumachen. Hierbei ist bemerkenswert, dass trotz deutlicher Zunahme an Untersuchungen der afrikanische Kontinent bislang keine Beachtung findet. Deisenhammer konnte keine spezifische Wettervariable ausmachen, die mit einem grundsätzlich höheren Suizidrisiko einhergeht, und führt dies auf die hohe Varianz der methodologischen Ansätze der eingeschlossenen Studien zurück [13]. In der vorliegenden Übersichtsarbeit erlaubt die nach wie vor äußerst heterogene Methodologie der erfassten Studien ebenso lediglich eine narrative Synthese ohne Metaanalyse. Obgleich sich zu allen meteorologischen Parametern inkonsistente und mitunter widersprüchliche Ergebnisse finden, stechen nunmehr die positiven Assoziationen zwischen Temperatur und Suizidalität in der vorliegenden Übersichtsarbeit deutlich hervor. Dass sich lediglich in 17 der insgesamt 99 eingeschlossenen Untersuchungen keine signifikanten Assoziationen zeigten, könnte gleichsam ein Hinweis auf ein Publikationsbias sein. Überdies berücksichtigen die

Nervenarzt 2020 91:227-232 https://doi.org/10.1007/s00115-019-00795-x

(c) Der/die Autor(en) 2019

C. Pervilhac · K. Schoilew $\cdot$ H. Znoj · T. J. Müller

\section{Wetter und Suizid. Assoziation zwischen meteorologischen Variablen und suizidalem Verhalten - eine qualitative systematische Übersichtsarbeit}

\section{Zusammenfassung}

Hintergrund. Die bereits in Erscheinung tretenden sowie künftig zu erwartenden Auswirkungen des globalen Klimawandels gehen mit weitreichenden Implikationen für die menschliche Gesundheit einher. In diesem Kontext rückt der Einfluss einzelner meteorologischer Variablen zunehmend in den Fokus der Suizidforschung.

Ziel der Arbeit. Systematische Übersichtsarbeit mit qualitativer Synthese der aktuellen Literatur zu Assoziationen zwischen Wettervariablen und Suizidversuchen sowie Suiziden.

Material und Methoden. Kriterienbasierte, systematische Literaturrecherche nach den PRISMA-Kriterien. Es wurden Originalarbeiten mit Peer-Review ohne zeitliche Beschränkung eingeschlossen.

Ergebnisse und Diskussion. Insgesamt 99 Studien wurden eingeschlossen und dem jeweiligen Studiendesign entsprechend auf Basis von Tages-, Wochen-, Monats- und Jahresdaten zusammengefasst. Die Mehrzahl der Studien zeigte signifikante Assoziationen mit mindestens einer Wettervariable, wobei inkonsistente und widersprüchliche Ergebnisse festzustellen waren. Am häufigsten fanden sich positive Korrelationen zwischen Temperatur und Suizidalität. Es zeigte sich eine hohe Heterogenität in Bezug auf die verwendeten Studiendesigns. Trotz weiterhin bestehendem Forschungs- und Klärungsbedarf werden in diesem Kontext ggf. sinnvolle Anknüpfungspunkte für die Suizidprävention offenbar.

Schlüsselwörter

Suizidalität · Meteorologische Faktoren . Temperatur - Psychische Gesundheit . Klimawandel

\section{Weather and suicide. Association between meteorological variables and suicidal behavior - a systematic qualitative review article}

\section{Abstract}

Background. The effects of current and expected future climate change on mental health outcomes are of increasing concern. In this context, the importance of meteorological factors on suicidal behavior is receiving growing attention in research.

Objective. Systematic review article with qualitative synthesis of the currently available literature, looking at the association between meteorological variables and attempted and completed suicide.

Material and methods. Criteria-based, systematic literature search according to the PRISMA criteria. Peer-reviewed original research studies were included without time limits.

Results and conclusion. A total of 99 studies were included and grouped according to the research analysis based on daily, weekly, monthly and annual data. The majority of the studies reported a statistical association with at least one meteorological variable. The most consistent positive correlation was shown between temperature and suicidal behavior. However, the results are not conclusive and in part contradictory. The reported studies differed distinctively in terms of study design. Meteorological parameters may be associated with suicidal behavior. Future research in this area is needed to provide further clarity. Despite existing knowledge gaps, the current findings may have implications for suicide prevention plans.

\section{Keywords}

Suicidality - Meteorological factors .

Temperature $\cdot$ Mental health $\cdot$ Climate change 
Hier steht eine Anzeige. Springer meisten Studien mehrere meteorologische Variablen. Informationen über eine Adjustierung des Signifikanzniveaus bei multiplem Testen sind jedoch nicht konsequent aufgeführt. Statistische Fehlschlüsse sind daher infolge von a-Fehler-Kumulierung mitunter nicht auszuschließen.

Dennoch sind die vorliegenden Ergebnisse hinweisgebend darauf, dass eine erhöhte Suizidalität weniger - wie gemeinhin angenommen - mit dunklem Regenwetter, sondern eher mit warmer/heißer Witterung assoziiert zu sein scheint.

\section{Erklärungsansätze}

In der Literatur wird speziell der Einfluss von Temperatur auf das psychische Wohlbefinden diskutiert. Im Rahmen einer rezenten systematischen Übersichtsarbeit wurde der Einfluss von Temperatur und Hitze auf die mentale Gesundheit bestätigt - die deutlichsten Effekte zeigten sich auch hierbei in einem erhöhten Suizidrisiko [50]. An extrem heißen Tagen wurde überdies ein Anstieg psychiatrischer Patienten (u.a. bipolare Störung, Schizophrenie, Demenz) in Notaufnahmen festgestellt [21, 50, 52, 54]. Zugrunde liegende biologische Mechanismen zum Einfluss meteorologischer Faktoren auf psychische Erkrankungen sind bisher weitgehend unbekannt. Patienten mit psychotischen Störungen scheinen per se wärmeempfindlich zu sein. So zeigten Daten von Shiloh et al. [47] sowie Chong und Castle [8], dass Schizophreniepatienten eine veränderte Thermoregulation aufweisen. Darüber hinaus erhöht eine Reihe psychopharmakologischer Medikamente die psychische Empfindlichkeit bei psychiatrischen Patienten [23, 29, 48], wobei ein zusätzlicher Alkoholkonsum diesen Effekt verstärkt [12]. Im Blick auf die Ätiopathogenese von Suizid beruhen die meisten Erklärungsansätze auf dem Diathese-Stress-Modell. Demnach werden Vulnerabilitätsfaktoren (Diathese) angenommen, die durch externe Stressoren bei prädisponierten Personen aktiviert werden und zu suizidalen Verhaltensweisen führen können [28, 37, 46]. Neben biopsychosozialen Faktoren finden zunehmend weitere Umweltfaktoren Berücksichtigung. In diesem Zusammenhang könnten Wettervariablen auf vielfältige Weise Einfluss auf das multifaktorielle Phänomen Suizidalität haben. So geht etwa nächtliche Hitze mit einer reduzierten Schlafqualität einher [34, 39] - dies kann sich bekanntermaßen negativ auf die individuelle mentale Gesundheit auswirken [2, 45]. Nach einer Analyse von über 600 Mio. Nachrichten auf der Internetplattform Twitter, wiesen Tweets in überdurchschnittlich heißen Monaten signifikant häufiger depressiv konnotierte Signalwörter auf [6]. Zusätzlich zu einzelnen meteorologischen Stressoren wird davon ausgegangen, dass der Klimawandel sowohl kurz- als auch langfristig auf unterschiedlichen Ebenen die psychische Gesundheit negativ beeinflussen kann [2, 3, 17, 19, 42]. So wird etwa mit Erhöhung der mittleren Monatstemperatur um $1^{\circ} \mathrm{C}$ eine Zunahme der Suizidrate in den USA um $0,7 \%$ und in Mexiko um $2,1 \%$ prognostiziert [6]. Es wird mit 9000 bis 40.000 zusätzlichen Suiziden infolge des Klimawandels bis 2050 in den USA und Mexiko ausgegangen [6].

\section{Limitationen und künftiger Forschungsbedarf}

Die prognostischen Daten unterstreichen die hohe gesellschaftspolitische Relevanz der Thematik und vergegenwärtigen den Forschungsbedarf in diesem Zusammenhang. Obgleich sich eine Ausweitung der Suizidforschung in diesem Bereich feststellen lässt, ist die Aussagekraft der Studienergebnisse aufgrund methodologischer Mängel nach wie vor limitiert. Grundsätzlich finden sich auf dem Gebiet ausschließlich ökologische Studien, in denen lediglich Assoziationen gemessen werden und sich keine kausalen Zusammenhänge nachweisen lassen. Überdies ist zu berücksichtigen, dass die Korrelationsanalysen auf Aggregatdaten basieren und keine Schlussfolgerung auf Individualebene erlauben: Assoziationen auf aggregierter Ebene sind nicht zwangsläufig gleichbedeutend mit Assoziationen auf individueller Ebene, es besteht somit das Risiko, falsche Rückschlusse von der Aggregatebene auf die Individualebene 
zu ziehen („ökologischer Fehlschluss“, „cross-level bias").

Künftige Forschungsbemühungen sollten auf konsistenter Erfassung der relevanten Variablen mit einer möglichst geringen zeitlichen und räumlichen Auflösung beruhen. Auswertungen auf Basis von Tagesdaten erscheinen hierbei aussagekräftiger als etwa Auswertungen, die auf mittleren Jahresdaten beruhen. Einerseits ermöglichen Untersuchungen auf Tagesbasis spezifischere Betrachtungen zur Bewertung meteorologischer Variablen im Sinne akuter Stressoren im Zusammenhang mit suizidalem Verhalten. Andererseits erlauben sie bei zusätzlicher Berechnung von Clusterund Lag-Effekten unter Umständen eine Betrachtung chronisch-additiver Effekte. In diesem Zusammenhang setzt eine aussagekräftige statistische Auswertung eine multidisziplinäre Expertise voraus, die unter anderem Epidemiologie, Statistik und Klimawissenschaften vereint: Vicedo-Cabrera et al. [53] geben hierzu etwa ein detailliertes „hands-on tutorial“ $\mathrm{zu}$ komplexen statistischen Verfahren, die dem aktuellen Stand der Wissenschaft entsprechen.

Darüber hinaus sollten - neben der Untersuchung von Risikoindikatoren mögliche Resilienzfaktoren und Adaptationsstrategien in der Forschung Berücksichtigung finden.

Innovative interdisziplinäre Forschungsansätze zum komplexen Einfluss geopolitischer, sozioökonomischer, ökologischer Faktoren sowie Umweltfaktoren auf die mentale Gesundheit im Zusammenhang mit Wetter und Klimawandel werden etwa durch Berry et al. beschrieben [4].

\section{Fazit für die Praxis}

Zusammenfassend zeigen sich Hinweise, dass meteorologische Faktoren mit suizidalem Verhalten assoziiert sein können. Insbesondere erhöhte Temperatur und Hitze scheinen mit einem höheren Suizidrisiko einherzugehen. Die bisweilen widersprüchlichen Ergebnisse zeugen von einem weiterhin bestehenden Forschungs- und Klärungsbedarf. Angesichts einer zu erwartenden $\mathrm{Zu}$ nahme von Wetterextremen (z. B. Hitze- wellen) infolge des globalen Klimawandels geht die Thematik mit weitreichenden Implikationen einher. Ausgehend davon könnte sich bereits jetzt eine Integration einfacher Maßnahmen in die Suizidprävention als sinnvoll erweisen. Entsprechende Präventions- und Adaptationsmaßnahmen sollten sowohl auf Individual- (u.a. Psychoedukation vulnerabler Gruppen, behaviorale Interventionen) als auch Kollektivebene (u. a. Schulungen im Gesundheitswesen, Sensibilisierung von Gesundheitspersonal, Warnsysteme bei drohender Hitze) Berücksichtigung finden.

\section{Korrespondenzadresse}

Prof. Dr. Thomas J. Müller

Privatklinik Meiringen, Willigen

3860 Meiringen, Schweiz

thomas.mueller@upd.unibe.ch

\section{Einhaltung ethischer Richtlinien}

Interessenkonflikt. C. Pervilhac, K. Schoilew, H. Znoj und T.J. Müller geben an, dass kein Interessenkonflikt besteht.

Für diesen Beitrag wurden von den Autoren keine Studien an Menschen oder Tieren durchgeführt. Für die aufgeführten Studien gelten die jeweils dort angegebenen ethischen Richtlinien.

Open Access Dieser Artikel wird unter der Creative Commons Namensnennung 4.0 International Lizenz (http://creativecommons.org/licenses/by/4.0/deed. de) veröffentlicht, welche die Nutzung, Vervielfältigung, Bearbeitung, Verbreitung und Wiedergabe in jeglichem Medium und Format erlaubt, sofern Sie den/die ursprünglichen Autor(en) und die Quelle ordnungsgemäßnennen, einen Linkzur Creative Commons Lizenz beifügen und angeben, ob Änderungen vorgenommen wurden.

\section{Literatur}

1. Ajdacic-Gross V, Bopp M, Ring M et al (2010) Seasonality in suicide-a review and search of new concepts for explaining the heterogeneous phenomena. Soc Sci Med 71:657-666

2. Berry HL, Bowen K, Kjellstrom T (2010) Climate change and mental health: a causal pathways framework. Int JPublic Health 55:123-132

3. Berry HL, Hogan A, Owen J et al (2011) Climate change and farmers' mental health: risks and responses. Asia Pac J Public Health 23:119S-1132

4. Berry HL, Waite TD, Dear KBG et al (2018) The case for systems thinking about climate change and mental health. Nat Clim Chang 8:282-290

5. Besancon F, Ricome H, Pezzi-Girault D et al (1981) Weather conditions in myocardial infarction, cerebrovascular strokes and suicide attempts. Sem Hop 57:1609-1611
6. Burke M, González F, Baylis P et al (2018) Higher temperatures increase suicide rates in the united states and mexico. Nat Clim Chang 8:723-729

7. Chiu LP (1988) Do weather, day of the week, and address affect the rate of attempted suicide in hong kong? Soc Psychiatry Psychiatr Epidemiol 23:229-235

8. Chong TW, Castle DJ (2004) Layer upon layer: thermoregulation in schizophrenia. Schizophr Res 69:149-157

9. Christodoulou C, Douzenis A, Papadopoulos FC et al (2012) Suicide and seasonality. Acta Psychiatr Scand 125:127-146

10. Coimbra DG, Pereira ESAC, De Sousa-Rodrigues CF et al (2016) Do suicide attempts occur more frequently in the spring too? A systematic review and rhythmic analysis. J Affect Disord 196:125-137

11. Costello A, Abbas M, Allen A et al (2009) Managing the health effects of climate change: Lancet and university college london institute for global health commission. Lancet 373:1693-1733

12. CusackL, De Crespigny C, Athanasos P (2011) Heatwaves and their impact on people with alcohol, drug and mental health conditions: a discussion paper on clinical practice considerations. J Adv Nurs 67:915-922

13. Deisenhammer EA (2003) Weather and suicide: the present state of knowledge on the association of meteorological factors with suicidal behaviour. Acta Psychiatr Scand 108:402-409

14. Dexter EG (1904) Weather influences: an empirical study of the mental and physiological effects of definite meteorological conditions. The Macmillan Company, New York

15. Dixon KW, Shulman MD (1983) A statistical investigation into the relationship between meteorological parameters and suicide. Int J Biometeorol 27:93-105

16. Dixon PG, Mcdonald AN, Scheitlin KN et al (2007) Effects of temperature variation on suicide in five u.S. Counties, 1991-2001. Int J Biometeorol 51:395-403

17. Doherty TJ, Clayton S (2011) The psychological impacts of global climate change. Am Psychol 66:265-276

18. Fernandez-Nino JA, Florez-Garcia VA, AstudilloGarcia Cl et al (2018) Weather and suicide: a decade analysis in the five largest capital cities of colombia. Int JEnviron Res Public Health 15:1313

19. Fritze JG, Blashki GA, Burke $S$ et al (2008) Hope, despair and transformation: climate change and the promotion of mental health and wellbeing. Int JMent Health Syst 2:13

20. Grove O, Lynge J (1979) Suicide and attempted suicide in greenland. A controlled study in nuuk (godthaab). Acta Psychiatr Scand 60:375-391

21. Hansen A, Bi P, Nitschke $M$ et al (2008) The effect of heat waves on mental health in a temperate australian city. Environ Health Perspect 116:1369-1375

22. Helama S, Holopainen J, Partonen T (2013) Temperature-associated suicide mortality: Contrasting roles of climatic warming and the suicide prevention program in finland. Environ Health Prev Med 18:349-355

23. Hermesh H, Shiloh R, Epstein Y et al (2000) Heat intolerance in patients with chronic schizophrenia maintained with antipsychotic drugs. Am J Psychiatry 157:1327-1329

24. Hiltunen L, Haukka J, Ruuhela R et al (2014) Local daily temperatures, thermal seasons, and suicide rates in finland from 1974 to 2010 . Environ Health Prev Med 19:286-294 
25. Larcan A, Martin J, Lambert H et al (1976) Suicidal behavior and meterological conditions. Ann Med Psychol (Paris) 1:51-61

26. Lester D (1999) Climatic data and national suicide and homicide rates. Percept Mot Skills 89:1036

27. Likhvar V, Honda Y, Ono M (2011) Relation between temperature and suicide mortality in japan in the presence of other confounding factors using timeseries analysis with a semiparametric approach. Environ Health Prev Med 16:36-43

28. Mann JJ, Waternaux C, Haas GL et al (1999) Toward a clinical model of suicidal behavior in psychiatric patients. Am J Psychiatry 156:181-189

29. Martin-Latry K, Goumy MP, Latry P et al (2007) Psychotropic drugs use and risk of heat-related hospitalisation. Eur Psychiatry 22:335-338

30. Mcmichael A, Berry H, Butler C (2014) Climate change. Ipccimpacts report: Staring into the abyss. World Nutr 5:432-435

31. Mcmichael AJ (2012) Insights from past millennia into climatic impacts on human health and survival. Proc Natl Acad Sci USA 109:4730-4737

32. Moher D, Liberati A, Tetzlaff J et al (2009) Preferred reporting items for systematic reviews and meta-analyses: the prisma statement. PLoS Med 6:e1000097

33. Morali D, Jehel L, Paterniti S (2008) The august 2003 heat wave in france: effects on psychiatric disorders and suicidal behavior. Presse Med 37:224-228

34. Mullins J, White C (2018) Temperature, climate change, and mental health: Evidence from the spectrum of mental health outcomes. Working Papers 1801. Polytechnic State University, Department of Economics, California

35. Nakaji S, Parodi S, Fontana V et al (2004) Seasonal changes in mortality rates from main causes of death in japan (1970-1999). Eur J Epidemiol 19:905-913

36. Nejar KA, Bensenor IM, Lotufo PA (2007) Sunshine and suicide at the tropic of capricorn, sao paulo, brazil, 1996-2004. Rev Saude Publica 41:1062-1064

37. O'connor RC, Nock MK (2014) The psychology of suicidal behaviour. Lancet Psychiatry 1:73-85

38. Obersteg Jl, Marzetta B (1957) The question of suicide and weather. Dtsch Z Gesamte Gerichtl Med 46:18-26

39. Obradovich N (2017) Climate change may speed democratic turnover. Clim Change 140:135-147

40. Partonen T, Haukka J, Viilo Ket al (2004) Cyclic time patterns of death from suicide in northern finland. JAffect Disord 78:11-19

41. Patscheider H (1958) Föhn und Selbstmord. Dtsch Z Gesamte Gerichtl Med 47:271-275

42. Patz JA, Frumkin H, Holloway Tet al (2014) Climate change: challenges and opportunities for global health. JAMA 312:1565-1580

43. Pokorny AD (1966) Suicide and weather. Effect of barometric pressure and humidity. Arch Environ Health 13:255-256

44. Pokorny AD, Davis F, Harberson W (1963) Suicide, suicide attempts, and weather. Am J Psychiatry 120:377-381

45. Robotham D (2011) Sleep as a public health concern: Insomnia and mental health. J Public Ment Health 10:234-237

46. Schotte DE, Clum GA (1987) Problem-solving skills in suicidal psychiatric patients. J Consult Clin Psychol 55:49

47. Shiloh R, Shapira A, Potchter O et al (2005) Effects of climate on admission rates of schizophrenia patients to psychiatric hospitals. Eur Psychiatry 20:61-64
48. Stollberger C, Lutz W, Finsterer J (2009) Heatrelated side-effects of neurological and nonneurological medication may increase heatwave fatalities. Eur J Neurol 16:879-882

49. Talaei A, Hedjazi A, Ardani AR et al (2014) The relationship between meteorological conditions and homicide, suicide, rage, and psychiatric hospitalization. J Forensic Sci 59:1397-1402

50. Thompson R, Hornigold R, Page L et al (2018) Associations between high ambient temperatures and heat waves with mental health outcomes: a systematic review. Public Health 161:171-191

51. Tsai JF, Cho W (2011) The secular trend of suicide rate and the socio-economic, media, and climatic factors in taiwan, 1976-2009: a population-based study. J Affect Disord 129:270-274

52. Vaneckova P, Bambrick H (2013) Cause-specific hospital admissions on hot days in sydney, australia. PLoSONE 8:e55459

53. Vicedo-Cabrera AM, Sera F, Gasparrini A (2019) Hands-on tutorial on a modeling framework for projections of climate change impacts on health Epidemiology 30:321-329

54. Wang X, Lavigne E, Ouellette-Kuntz H et al (2014) Acute impacts of extreme temperature exposure on emergency room admissions related to mental and behavior disorders in toronto, canada. J Affect Disord 155:154-161

55. White RA, Azrael D, Papadopoulos FC et al (2015) Does suicide have a stronger association with seasonality than sunlight? BMJ Open 5:e7403

56. World Health Organization (2018) Suicide: Key facts. https://www.who.int/news-room/factsheets/detail/suicide. Zugegriffen: 17. Jan. 2019

57. Zung WW, Green RL Jr. (1974) Seasonal variation of suicide and depression. Arch Gen Psychiatry 30:89-91

\section{Vergleichsstudie geht Fibro- myalgie-Syndrom auf den Grund}

Menschen mit einem Fibromyalgie-Syndrom (FMS) leben mit starken chronischen Schmerzen am gesamten Körper. Vor allem Frauen sind betroffen. Früher als Weichteilrheuma bezeichnet, konnte vor wenigen Jahren nachgewiesen werden, dass dem Syndrom eine Schädigung des Nervensystems zugrundeliegt, die einen muskulären Schmerz verursacht. Ein erster Nachweis zum besseren Verständnis war erbracht, die Ursachen aber noch nicht gefunden. Wissenschaftler der Klinik für Psychosomatische Medizin und Psychotherapie im LWLUniversitätsklinikum der Ruhr-Universität Bochum suchen derzeit für ihre Vergleichsstudie gesunde Frauen, die mit Hilfe einer einfachen Mundspülung einen Beitrag leisten können, Hinweise zur Entstehung des Fibromyalgie-Syndroms zu finden. Prof. Dr. Martin Diers, zuständig für die Klinische und Experimentelle Verhaltensmedizin in der LWL-Klinik für Psychosomatische Medizin und Psychotherapie, benötigt für seine Studie dringend gesunde Probandinnen zwischen 40 und 60 Jahren: „Gerade bei Vergleichsstudien sind wir auf die Daten von Gesunden und Kranken angewiesen, damit wir diese gegenüberstellen und Unterschiede generieren können. Nur so können wir ein genaues Krankheitsbild von Schmerz-Syndromen erfassen und dann gezielt Behandlungsfelder schaffen." Viele Menschen, die unter chronischen Schmerzen leiden, erhalten nicht die richtige Behandlung, da es diese oftmals noch gar nicht gibt. Um Betroffene angemessen therapieren zu können, ist es daher zunächst wichtig, die Schmerzursache zu ermitteln und zu verstehen, wieso ein chronischer Schmerz überhaupt erst entsteht. Die FMS-Studie befasst sich mit der Erforschung molekulargenetischer Faktoren. Das heißt, mit Hilfe der über eine Mundspülung erfassten DNA werden die relevanten Erbinformationen von gesunden und kranken Teilnehmern verglichen. Im Rahmen eines Fragebogens werden zusätzlich Informationen zur psychischen und körperlichen Befindlichkeit gesammelt und den DNA-Daten gegenübergestellt. 